\title{
Dynamic and Static Pricing Strategies with Reference Quality Effect in Closed- Loop Supply Chain
}

\author{
Zhihchao Zhang ${ }^{1}$, Mingdong Wan ${ }^{1}$, Fang Chen ${ }^{1}$, Xingxing Tang ${ }^{1}$, Zhi Liu ${ }^{2}$ * \\ ${ }^{1}$ College of management, Anhui Science and Technology University, Anhui 233100, PR China \\ ${ }^{2}$ Odette School of Business, University of Windsor, Windsor, Ontario N9B 3P4, Canada \\ ekmzzc@163.com
}

Keywords: dynamic pricing strategy; differential game; reference quality effects; CLSC

\begin{abstract}
With the rapid development of e-commerce and technology, dynamic pricing, as an effective way to enhance company profits, has been widely adopted by firms worldwide. However, there are little researches on the effects of dynamic pricing on closed-loop supply chain (CLSC). The paper considers a Stackelberg game between one manufacturer and one retailer where the manufacturer, as the leader, decides the product quality and the wholesale price, and the retailer, as the follower, sets the retail price. Through the analysis of the research, it is concluded the following results: It is best for the manufacturer, the retailer and the whole supply chain to adopt dynamic pricing strategies compared with static pricing strategies in both two kinds of cases. In dynamic pricing strategies, the manufacturer should take a higher recycling rate where the recovery cost is low while take a lower recycling ratio where the recovery
\end{abstract} cost is high.

\section{INTRODUCTION}

With the development of technology and economic progress, people's pursuit of products has gone from price to a diversified pursuit of quality, price and environmental protection. Therefore, enterprises need to take a comprehensive consideration of the price, quality and environmental protection of the products in the decision-making process to get better progress. The price influencing demand and reflecting its quality directly, is one of the crucial product elements that the customers value. Quality is the life of the product, especially in a fiercely competitive market environment, enterprises must improve the quality of the products to meet the increasing quality demand of consumer for long-term blossom. With the improvement of people's living standard, the awareness of environmental protection also increased, at the same time, in many countries, government make some environmental policies to strengthen disposal of the waste, therefore, enterprise implement re-manufacturing partly due to comply with environmental legislation and partly due to the potential profit generated by sustainable productions.

"Quality first, quality is life", these slogans are often found in the companys' quality policy which indicate that quality is the source of product vitality has been widely recognized among a large proportion of firms. Quality as a key factor to affect product price, sales and other market conditions was presented in reality. Two constructive contracts were investigated by product recycling costs shared between a manufacturer and a supplier to stimulate quality improvement effort (Chao et al, 2009). A risk-averse supply chain combined with quality investment efforts and price decision under uncertain demand has been reseached (Xie et al, 2011). A recent study exploreed wholesale price and revenuesharing contracts in terms of their performance and coordination in a two-echelon supply chain (EI Quardighi and Kogan, 2013).

Price representing the enterprise market position and reflecting its response to the market directly, is the approach that divides customer groups into several parts. Enterprise's price position is not steady, but as changing with the market environment, product life cycle and enterprise progress stage. Dynamic adjustment of prices well reflects the change of market and customer needs. It obtains additional insights into the relative effects of price and quality by relaxing the structured dependency between quality and price(Gavious and Lowengart, 2012). It is demonstrated that revenue-sharing contract coordinates the supply chain consisting of one supplier and one retailer, and arbitrarily divides the resulting profits for any reasonable revenue function that depends on the retailers purchase quantity and price(Cachon and Lariviere, 2005).

The implementation of closed-loop supply chain can not only respond to the government's related environmental protection policies, but also save resource and decrease the loss of the company. It is more advantageous to cut down the production cost, raise the profit of the enterprise and enhance the overall image of the enterprise. Recent operations management literature on closed-loop supply chains has highlighted the significance of product take-back and economic advantages associated with re-manufacturing or recycling end-of-use products.

However, to our best knowledge, there are no literature researching the dynamic pricing strategies with reference quality effects in closed- loop supply chain.

\section{PROBLEM DEFINITION}

Constructing a supply chain composed of one manufacturer (M) and one retailer(R). We consider a Stackelberg game between the manufacturer, who as the 
leader decides the wholesale price ( $w$ ) and quality strategy $\left({ }^{q}\right)$, and the retailer, who as the follower determines the retail price (p). The manufacturer produces the new prroducts as well as the remanufacturing items, and the cost of the new product is $C_{m}$ while ${ }^{C_{\mathrm{r}}}$ represents the cost of remanufacturing goods.

Consider a finite planning horizon $[0, T]$, and let $R(t)$ denotes the goodwill and $q(t)$ express the current quality of the products. According to (Guowei Liua, et al., 2015), we use an exponential smoothing process of historical quality to model the dynamics of reference quality as follows:

$$
\dot{R}(t)=\theta(q(t)-R(t)), R(0)=R_{0}
$$

Where $\theta>0$ captures the depreciation of the goodwill stock, and $R_{0}>0$ denotes the initial stock of goodwill.

We assume that the demand $D(\mathrm{t})$ is dependent on price, quality and the difference between product quality and reference quality, and adopt the following demand function

$$
D(t)=\alpha-\beta p(t)+\gamma_{1} q(t)+\gamma_{2}(q(t)-R(t))
$$

Where $\alpha>0$ represents the basic market capacity, $0 \leq \beta \leq 1$ denotes the price discount coefficient.

In accordance with previous literature(Feng et al. 2014), the quality cost function is assumed to be quadratic in the quality rate:

$$
C(q)=\frac{1}{2} k_{1} q^{2}(t)
$$

According to previous research (Guide and Van Wassenhove 2009), the product recycled cost function is assumed to be quadratic in the recycle rate:

$$
C(\tau)=\frac{1}{2} k_{2} \tau^{2}
$$

where $k_{1}>0$ is a measure value of manufacture cost, and $k_{2}>0$ is a measure value of recycle cost.

The objective functions of the manufacturer and retailer are expressed respectively asfollows:

$$
\begin{gathered}
J_{M}=\int_{0}^{T}\left(\left(w-c_{m}+\Delta \tau\right) D(t)-\frac{1}{2} k_{1} q^{2}(t)-\frac{1}{2} k_{2} \tau^{2}\right) d t \\
J_{R}=\int_{0}^{T}((p-w) D(t)) d t
\end{gathered}
$$

Taking the dynamic relationships (1)- (6) together, the differential game is presented as follows:

$$
\begin{aligned}
& \max _{w(\cdot), q(\cdot)} J_{M}=\int_{0}^{T}\left(\left(w-c_{m}+\Delta \tau\right) D(t)-\frac{1}{2} k_{1} q^{2}(t)-\frac{1}{2} k_{2} \tau^{2}\right) d t \\
& \max _{p(\cdot)} J_{R}=\int_{0}^{T}((p-w) D(t)) d t \\
& \text { s.t. } \dot{R}(t)=\theta(q(t)-R(t)), R(0)=R_{0}
\end{aligned}
$$

\section{SOLUTIONS}

In this section, we consider the decentralized scenario where the manufacturer plays a Stackelberg game as model (7). The equilibrium pricing and quality strategies are presented in two cases caused by different pricing strategies, dynamically or statically. To promote understanding, we use the superscripts " ss" and "dd" to signify "the static wholesale price and retail price case" and "the dynamic wholesale price and retail price case", respectively.

\subsection{The static wholesale price and static retail price case}

Solving the differential game (7), we can get the equilibrium pricing and quality strategies in the case where both the manufacturer and the retailer adopt a static pricing strategy as the following proposition.

Proposition1. In the static wholesale price and static retail price case, the equilibrium pricing strategies for both the manufacturer and the retailer, and the equilibrium quality strategy are showed:

$$
\begin{gathered}
w^{s s}=\frac{2\left(\alpha+\left(c_{m}-\Delta \tau\right) \beta\right) \theta k_{1} T}{4 \beta k_{1} \theta T+M} \\
+\frac{2 R_{0} \gamma_{2} k_{1}\left(e^{-\theta T}-1\right)-\left(\Delta \tau-c_{m}\right) M}{4 \beta k_{1} \theta T+M} \\
p^{s s}=\frac{\left(\alpha+\left(\Delta \tau-c_{m}\right) \beta\right) \theta T+R_{0} \gamma_{2}\left(e^{-\theta T}-1\right)}{8 \beta^{2} k_{1} \theta T^{2}+2 \beta T M} C+ \\
\frac{\alpha}{2 \beta}+\frac{\gamma_{2} R_{0}\left(e^{-\theta T}-1\right)}{2 \beta T \theta}-\frac{2\left(\alpha-\left(\Delta \tau-c_{m}\right) \beta\right) \theta k_{1} T}{8 \beta k_{1} \theta T+2 M} \\
+\frac{\left(\Delta \tau-c_{m}\right) M-2 R_{0} \gamma_{2} k_{1}\left(e^{-\theta T}-1\right)}{8 \beta k_{1} \theta T+2 M}
\end{gathered}
$$

Where

$$
\begin{array}{r}
C_{1}=\frac{2 T \theta \gamma_{1}^{2}+4\left(1-e^{-\theta T}\right) \gamma_{1} \gamma_{2}+\left(1-e^{-\theta T}\right) \gamma_{2}^{2}}{2 \theta} \\
q^{s s}=\frac{\left(\alpha+\left(\Delta \tau-c_{m}\right) \beta\right) \theta T+R_{0} \gamma_{2}\left(e^{-\theta T}-1\right)}{4 \beta k_{1} \theta T+M}\left(\gamma_{2} e^{\theta(t-T)}+\gamma_{1}\right)
\end{array}
$$

And the corresponding goodwill is

$$
\begin{aligned}
& R^{s s}(t)=R_{0} e^{-\theta t}+\frac{\left(\alpha+\left(\Delta \tau-c_{m}\right) \beta\right) \theta T+R_{0} \gamma_{2}\left(e^{-\theta T}-1\right)}{4 \beta k_{1} \theta T+M} \\
& {\left[\gamma_{1}\left(1-e^{-\theta t}\right)+\frac{\gamma_{2}}{2}\left(e^{\theta(t-T)}-e^{-\theta(t+T)}\right)\right]}
\end{aligned}
$$

where $M$ and $C$ is denoted in Appendix A.

\subsection{The dynamic wholesale price and dynamic retail price case}

Solving the differential game (7) with static wholesale price and dynamic retail price, we can get the equilibrium pricing and quality strategies in the case where the manufacturer adopts a static wholesale price while the retailer adopts a dynamic retail price as the following proposition.

Proposition2. In the dynamic wholesale price and dynamic retail price case, the equilibrium pricing 
strategies for both the manufacturer and the retailer, and the equilibrium quality strategy are given by

$$
\begin{aligned}
& w_{M}^{d d}=\frac{2 k_{1}\left(\alpha+\beta\left(c_{m}-\Delta \tau\right)\right)+\left(\gamma_{1}+\gamma_{2}\right)^{2}\left(\Delta \tau-c_{m}\right)}{4 \beta k_{1}-\left(\gamma_{1}+\gamma_{2}\right)^{2}} \\
& -\frac{2 k_{1} \gamma_{2}}{4 \beta k_{1}-\left(\gamma_{1}+\gamma_{2}\right)^{2}}\left(h_{1} e^{r_{1} t}+h_{2} e^{r_{2} t}-B_{1}\right) \\
& +\frac{2 \beta \theta\left(\gamma_{1}+\gamma_{2}\right)}{4 \beta k_{1}-\left(\gamma_{1}+\gamma_{2}\right)^{2}}\left(h_{1} P_{1} e^{r_{1} t}+h_{2} P_{2} e^{r_{2} t}-A_{1}\right) \\
& q_{M}^{d d}=\frac{\left(\gamma_{1}+\gamma_{2}\right)\left(\alpha+\beta\left(\Delta \tau-c_{m}\right)\right)}{4 \beta k_{1}-\left(\gamma_{1}+\gamma_{2}\right)^{2}}- \\
& \quad \frac{\left(\gamma_{2}^{2}+\gamma_{1} \gamma_{2}\right)}{4 \beta k_{1}-\left(\gamma_{1}+\gamma_{2}\right)^{2}}\left(h_{1} e^{r_{1} t}+h_{2} e^{r_{2} t}-B_{1}\right) \\
& +\frac{4 \beta \theta}{4 \beta k_{1}-\left(\gamma_{1}+\gamma_{2}\right)^{2}}\left(h_{1} P_{1} e^{r_{1} t}+h_{2} P_{2} e^{r_{2} t}-A_{1}\right) \\
& p^{d d}=\frac{-3 k_{1} \gamma_{2}}{4 \beta k_{1}-\left(\gamma_{1}+\gamma_{2}\right)^{2}}\left(h_{1} e^{r_{1} t}+h_{2} e^{r_{2} t}-B_{1}\right) \\
& +\frac{3 \theta\left(\gamma_{1}+\gamma_{2}\right)}{4 \beta k_{1}-\left(\gamma_{1}+\gamma_{2}\right)^{2}}\left(h_{1} P_{1} e^{r_{1} t}+h_{2} P_{2} e^{r_{2} t}-A_{1}\right) \\
& +\frac{\left(k_{1} \beta-\left(\gamma_{1}+\gamma_{2}\right)^{2}\right)\left(c_{m}-\Delta \tau\right)+3 k_{1} \alpha}{4 \beta k_{1}-\left(\gamma_{1}+\gamma_{2}\right)^{2}}
\end{aligned}
$$

the corresponding goodwill is

$$
R^{d d}(t)=h_{1} e^{r_{1} t}+h_{2} e^{r_{2} t}-B_{1}
$$

where $h_{1}, h_{2}, r_{1}, r_{2}, A_{1}, B_{1}, N_{2}, P_{1}, P_{2}$ are showed in Appendix A.

\section{NUMERICAL STUDY}

In this part, we need numerical examples to show the effectiveness of results and assess the sensitivity analysis of some key parameters to examine their different influences on the optimal solutions.

Let $T=10$, and the following parameters setting: $\alpha=50, \beta=0.6, \gamma_{1}=0.7, \gamma_{2}=0.5, \theta=0.2$,

$R_{0}=10, k_{1}=15, k_{2}=12, c_{m}=10, c_{r}=8$;According to the proposition above and calculated in Matlab, we obtain

\begin{tabular}{|c|c|c|c|c|c|c|c|}
\hline & & $J^{S S}$ & $J^{d d}$ & $J_{M}^{S S}$ & $J_{M}^{d d}$ & $J_{R}^{S S}$ & $J_{R}^{d d}$ \\
\hline \multirow{3}{*}{$\beta$} & 0.2 & 3732.5 & 4060.6 & 2436.4 & 2664.5 & 1296.7 & 1396.6 \\
\hline & 0.4 & 1593.8 & 1755.7 & 1051.6 & 1161.8 & 542.75 & 594.53 \\
\hline & 0.6 & 943.88 & 1048.2 & 625.08 & 695.51 & 319.41 & 353.27 \\
\hline \multirow{5}{*}{$\theta$} & 0.8 & 632.38 & 707.56 & 419.52 & 470.14 & 213.40 & 237.96 \\
\hline & 0.2 & 943.88 & 1048.2 & 625.01 & 695.51 & 319.41 & 353.27 \\
\hline & 0.4 & 959.98 & 1078.2 & 636.36 & 716.13 & 324.16 & 362.61 \\
\hline & 0.6 & 993.30 & 1103.6 & 658.65 & 733.15 & 335.20 & 370.95 \\
\hline & 0.8 & 1025.6 & 1123.8 & 680.19 & 746.69 & 345.98 & 377.61 \\
\hline \multirow{3}{*}{$\gamma_{1}$} & 0.4 & 922.55 & 1027.8 & 613.57 & 684.20 & 309.52 & 344.13 \\
\hline & 0.5 & 928.83 & 1033.7 & 616.99 & 687.54 & 312.38 & 346.75 \\
\hline & 0.6 & 935.93 & 1040.6 & 620.80 & 691.30 & 315.67 & 349.79 \\
\hline \multirow{5}{*}{$\gamma_{2}$} & 0.7 & 943.88 & 1048.2 & 625.01 & 695.51 & 319.41 & 353.27 \\
\hline & 0.3 & 1064.6 & 1127.8 & 705.72 & 748.30 & 359.40 & 380.08 \\
\hline & 0.4 & 1003.8 & 1087.8 & 665.11 & 721.72 & 339.29 & 366.61 \\
\hline & 0.5 & 943.88 & 1048.2 & 625.01 & 695.51 & 319.41 & 353.27 \\
\hline & 0.6 & 884.66 & 1007.2 & 585.41 & 674.31 & 299.79 & 334.07 \\
\hline
\end{tabular}
the following profits for the manufacturer ,the retailer and the whole supply chain in two kinds of modes:

$$
\begin{aligned}
& J^{s s}=943.9, J^{d d}=1048.2, \quad J_{M}^{s s}=635.1, J_{M}^{d d}=695.5, \\
& J_{R}^{s s}=319.4, \quad J_{R}^{d d}=353.3 。
\end{aligned}
$$

Next, we assess the sensitivity analysis of some important parameters, such as $\beta, \theta, \gamma_{1}$ and, $\gamma_{2}$ to examine the impacts on profit for the manufacturer, the retailer and the supply chain in each case, which are shown in the table, respectively.

Table 1. Impacts on profits

According to the table, then we can get some results:

(1) The profits for the manufacturer, the retailer and the supply chain were declining as the value of $\beta$ and $\gamma_{2}$ increased.

(2) The profits for manufacturer, the retailer and the supply chain showed an upward trend as the value of $\theta$ and $\gamma_{1}$ increased.

Comparing dynamic and static pricing strategies, an apparent result is that the manufacturer,the retailer and the whole supply chain get a more profitable revenue with dynamic pricing than that with pricing statically, that is, $J^{d d}>J^{s s}, J_{M}^{d d}>J_{M}^{s s}, J_{R}^{d d}>J_{R}^{s s}$. It means that the dynamic pricing benefits the whole supply chain and its members.

Espically, we discuss the influence the recover fraction coefficient have on the whole supply chain and its members.

We can infer that Fig.1 vivily depicts that the manufacturer get minimum profit when not taking return 
policy, that is $\tau=0$, it means that the manufacturer takes the recycling policy will benifit more and gets a continuously increasing profit with a specific range of $k_{2}$ and a firstly increased and then decreased revenue with $k_{2}$ beyond that range.

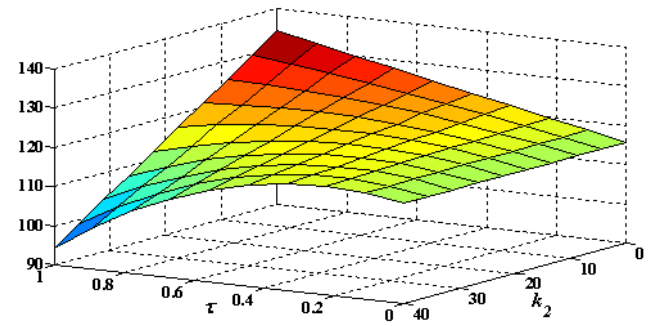

Fig. 1 The relationship between profit , ${ }^{\tau}$ and $k_{2}$

\section{CONCLUSIONS}

In this article, we consider a closed-loop supply chain consisting of a manufacturer and a retailer, and compare dynamic and static pricing strategies based on centralized and decentralized models. We construct a Stackelberg game where the manufacturer, as the leader, decides the product quality and the wholesale price, and the retailer, as the follower, sets the retail price. and apply difference equation to obtain the optimal solutions in all kinds of cases.

In addition, this article explores the sensitivity analysis of related parameters then concludes the following results: (1) For the manufacturer, the retailer and the entire supply chain, it is best for them to adopt dynamic pricing strategies compared with static pricing strategies both in integrated and decentralized case. (2) In dynamic pricing strategies, recycling fraction should be determined by recovery cost, the manufacturer should take a higher recycling rate where the recovery cost is low while should take a lower recycling ratio where the recovery cost is high.

\section{ACKNOWLEDGEMENTS}

This paper was supported by The Fourth Social Science and Pragmatic Measures in 2017 of Chuzhou, Anhui, China, No. A2017011.Talent Introduction Project of Anhui Science and Technology University: Research on supply chain coordination mechanism under asymmetric information.

\section{REFERENCES}

[1] Cachon, G. P., Lariviere, M. A., 2005. Supply chain coordination with revenue-sharing contracts: strengths and limitations. Management science, 51(1), 30-44.

[2] Chao, G. H., Iravani, S. M., Savaskan, R. C., 2009. Quality improvement incentives and product recall cost sharing contracts. Management Science,55(7), 1122-1138.

[3] Elmaghraby, W., Keskinocak, P., 2003. Dynamic pricing in the presence ofinventory considerations: Research overview, current practices, and futuredirections. Management Science,
49(10), 1287-1309.

[4] Feng, L., Zhang, J., Tang, W., 2015. A joint dynamic pricing and advertising model of perishable products. Journal of the Operational Research Society, 66(8), 1341-1351.

[5] Gavious, A., Lowengart, O., 2012. Price-quality relationship in the presence of asymmetric dynamic reference quality effects. Marketing Letters, 23(1), 137-161.

[6] Guide Jr, V. D. R., Van Wassenhove, L. N., 2009. OR FORUM-The evolution of closed-loop supply chain research. Operations research, 57(1), 10-18.

[7] Guo, J., Wen, Z., Zhou, Y., Ji, K., 2015. The competitive strategies between the traditional and online retailers. In Service Systems and Service Management (ICSSSM), 2015 12th International Conference on (pp. 1-5). IEEE.

[8] Xie, G., Yue, W., Wang, S., Lai, K. K., 2011. Quality investment and price decision in a risk-averse supply chain. European Journal of Operational Research, 214(2), 403410

\section{APPENDIX}

Since the manufacturer and the retailer emplied a Stackelberg game and the manufacturer is the leader, we first obtain the retailer's optimal reaction. The optimization problem for the retailer can be formulated as follows:

$$
\max _{p} \int_{0}^{T}\left\{(p-w)\left(\alpha-\beta p+\gamma_{1} q(t)+\gamma_{2}(q(t)-R(t))\right)\right\} d t
$$

Solving it, we can gain the optimal reaction $p^{\text {ss }}$ as below,

$$
p^{s s}=\frac{\int_{0}^{T}\left\{\alpha+\gamma_{1} q(t)+\gamma_{2}(q(t)-R(t))\right\} d t-w \beta T}{2 \beta T}
$$

Substituting $p^{\text {ss }}$ into the objective function of the manufacturer, we can get the Hamiltonian function of the manufacturer :

$$
\begin{aligned}
& H_{M}^{s s}=\left(w-c_{m}+\Delta \tau\right) \frac{\alpha+\gamma_{1} q+\gamma_{2}(q-R)-w \beta}{2} \\
& -\frac{k_{1}}{2} q^{2}+\lambda_{M}^{s s} \theta(q-R)
\end{aligned}
$$

Where $\lambda_{M}^{s s}$ is the adjoint variable associated with $R$, according to the first-order condition for optimality

$$
\begin{array}{r}
\frac{\partial H_{M}^{s s}}{\partial q}=0 \text { and adjoint equation } \begin{array}{r}
\lambda_{M}^{s s}=-\frac{\partial H_{M}^{s s}}{\partial R} \\
\text {, we can gain: } \\
q^{s s}=\frac{\left(\gamma_{1}+\gamma_{2}\right)\left(w-c_{m}+\Delta \tau\right) \theta \lambda_{M}^{s s}}{k_{1}} \\
\dot{\lambda}_{M}^{s s}=\frac{1}{2} \gamma_{2}\left(w-c_{m}+\Delta \tau\right)+\theta \lambda_{M}^{s s}
\end{array}
\end{array}
$$

Working out $\dot{\lambda}_{M}^{\text {ss }}$ with the terminal condition $\lambda_{M}^{\text {ss }}(T)=0$,we can obtain

$$
\lambda_{M}^{s s}=\frac{1}{2} \gamma_{2}\left(w-c_{m}+\Delta \tau\right)\left(e^{\theta(t-T)}-1\right)
$$




$$
q^{s s}=\frac{\left(w-c_{m}+\Delta \tau\right)\left(\gamma_{2} e^{\theta(t-T)}+\gamma_{1}\right)}{2 k_{1}}
$$

Substituting equation into $R(t)$ with the initial condition $R(0)=R_{0}$, we have

$$
\begin{aligned}
& R(t)=R_{0} e^{-\theta t}+\frac{\left(w-c_{m}+\Delta \tau\right)}{2 k_{1}}\left[\gamma_{1}\left(1-e^{-\theta t}\right)\right. \\
& \left.+\frac{\gamma_{2}}{2}\left(e^{\theta(t-T)}-e^{-\theta(t+T)}\right)\right]
\end{aligned}
$$

Next, we settle the optimal static wholesale price $w^{\text {ss }}$ of the manufacturer to the retailer. The optimization problem can be formulated as follows:

$$
\max _{w} \int_{0}^{T}\left\{\left(w-c_{m}+\Delta \tau\right)\left(\begin{array}{l}
\alpha-\beta p(t)+\gamma_{1} q(t) \\
+\gamma_{2}(q(t)-R(t))
\end{array}\right)-\frac{k_{1}}{2} q^{2}(t)\right\} d t
$$

Substitute Eqs., the maximization of the manufacturer provides the necessary condition for the static wholesale price as below:

$$
\begin{gathered}
w^{s s}=\frac{2\left(\alpha+\left(c_{m}-\Delta \tau\right) \beta\right) \theta k_{1} T-\left(\Delta \tau-c_{m}\right) M+2 R_{0} \gamma_{2} k_{1}\left(e^{-\theta T}-1\right)}{4 \beta k_{1} \theta T+M} \\
M=-\left(\theta \gamma_{1} \gamma_{2} T+\left(\gamma_{2}-\frac{1}{2} \gamma_{2}^{2}-1\right)\right)\left(e^{-\theta T}-1\right)-\frac{1}{4}\left[\gamma_{2}\left(1+e^{-\theta T}\right)\right. \\
\left.+4 \theta \gamma_{1} \gamma_{2}\right]\left(1-e^{-\theta T}+\frac{1}{2} \theta \gamma_{2}^{2} T\right)+\left(\gamma_{1}+\gamma_{2}\right)\left(2 \gamma_{1} \theta T+2 \gamma_{2}\left(1-e^{-\theta T}\right)\right)
\end{gathered}
$$

Substituting the optimal static wholesale price, we can get the equilibrium retail pricing strategy, quality strategy and the corresponding reference quality respectively respectively. The proof is complete.

\section{A2 Proof of Proposition 2}

We first derive the retailer's optimal reaction to gain Stackelberg equilibrium. Introducing an adjoint variable $\lambda_{R}^{\text {dd }}$ associated with the state variable $R$, we can obtain the Hamiltonian function of the retailer as follows:

$$
H_{R}^{d d}=(p-w)\left(\alpha-\beta p+\gamma_{1} q+\gamma_{2}(q-R)\right)+\lambda_{R}^{d d} \theta(q-R)
$$

According to the first-order condition $\frac{\partial H_{R}^{d d}}{\partial p^{d d}}=0$, the optimal reaction ${ }^{d d}$ can be given by

$$
p^{d d}=\frac{\alpha+\gamma_{1} q+\gamma_{2}(q-R)+\beta w}{2 \beta}
$$

Substituting $p^{d d}$ into the adjoint equation $\dot{\lambda_{R}^{d d}}=-\frac{\partial H_{R}^{d d}}{\partial R}$,we have

$$
\lambda_{R}^{\dot{d d}}=\frac{\alpha+\gamma_{1} q+\gamma_{2}(q-R)-\beta w}{2 \beta} \gamma_{2}+\theta \lambda_{R}^{\text {dd }}
$$

Then we get the Hamiltonian function of the manufacturer, which is given by

$$
\begin{aligned}
& H_{M}^{d d}=\left(w-c_{m}+\Delta \tau\right)\left(\alpha-\beta p+\gamma_{1} q+\gamma_{2}(q-R)\right) \\
& -\frac{k_{1}}{2} q^{2}-\frac{k_{2} \tau^{2}}{2}+\lambda_{M}^{d d} \theta(q-R)+\mu^{d d} \dot{\lambda_{R}^{d d}}
\end{aligned}
$$

where $\lambda_{M}^{\mathrm{dd}}$ and $\mu_{M}^{\mathrm{dd}}$ are the adjoint variables associated with $R$ and $\dot{\lambda}_{M}^{d d}$, respectively.

$$
\begin{aligned}
& H_{M}^{d d}=\left(w-c_{m}+\Delta \tau\right) \frac{\alpha+\gamma_{1} q+\gamma_{2}(q-R)-\beta w}{2}-\frac{k_{1}}{2} q^{2}-\frac{k_{2} \tau^{2}}{2} \\
& +\lambda_{M}^{d d} \theta(q-R)+\mu^{d d}\left(\frac{\alpha+\gamma_{1} q+\gamma_{2}(q-R)-\beta w}{2 \beta} \gamma_{2}+\theta \lambda_{R}^{d d}\right)
\end{aligned}
$$

According to the first-order condition for optimality

$$
\begin{gathered}
\frac{\partial H_{M}^{d d}}{\partial q}=0 \quad \frac{\partial H_{M}^{d d}}{\partial w}=0 \quad \text { and the adjoint equations } \\
\dot{\lambda_{M}^{d d}}=-\frac{\partial H_{M}^{d d}}{\partial R} \quad \dot{\mu_{M}^{d d}}=-\frac{\partial H_{M}^{d d}}{\partial \lambda_{R}^{d d}} \quad \text { we can get: } \\
w_{M}^{d d}=\frac{2\left(\gamma_{1}+\gamma_{2}\right)\left(2 \beta \theta \lambda_{M}^{d d}+\left(\Delta \tau \beta-c_{m} \beta+\gamma_{2} \mu\right)\left(\gamma_{1}+\gamma_{2}\right)\right)}{4 \beta^{2} k_{1}-\beta\left(\gamma_{1}+\gamma_{2}\right)^{2}} \\
+\frac{k_{1} \beta\left(\alpha+\beta\left(c_{m}-\Delta \tau\right)-\mu \gamma_{2}-\gamma_{2} R\right)}{4 \beta^{2} k_{1}-\beta\left(\gamma_{1}+\gamma_{2}\right)^{2}} \\
q_{M}^{d d}=\frac{\left(\gamma_{2}+\gamma_{1}\right)\left(\alpha+\beta \Delta \tau-\beta c_{m}\right)+4 \beta \theta \lambda_{M}^{d d}}{4 \beta k_{1}-\left(\gamma_{1}+\gamma_{2}\right)^{2}} \\
+\frac{\left(\gamma_{2}^{2}+\gamma_{1} \gamma_{2}\right)\left(\mu^{d d}-R\right)}{4 \beta k_{1}-\left(\gamma_{1}+\gamma_{2}\right)^{2}}
\end{gathered}
$$

According to the initial condition $\mu^{\text {sd }}(0)=0$, distinctly we can obtain:

$$
\mu^{s d} \equiv 0
$$

$$
\begin{array}{r}
q_{M}^{d d}=\frac{\left(\gamma_{1}+\gamma_{2}\right)\left(\alpha+\beta \Delta \tau-\beta c_{m}\right)+4 \beta \theta \lambda_{M}^{d d}-R\left(\gamma_{2}^{2}+\gamma_{2} \gamma_{1}\right)}{4 \beta k_{1}-\left(\gamma_{1}+\gamma_{2}\right)^{2}} \\
w_{M}^{d d}=\frac{\left(\gamma_{1}+\gamma_{2}\right)\left(2 \theta \lambda_{M}^{d d}+\left(\Delta \tau-c_{m}\right)\left(\gamma_{1}+\gamma_{2}\right)\right)}{4 \beta k_{1}-\left(\gamma_{1}+\gamma_{2}\right)^{2}} \\
+\frac{2 k_{1}\left(\alpha+\beta\left(c_{m}-\Delta \tau\right)-\gamma_{2} R\right)}{4 \beta k_{1}-\left(\gamma_{1}+\gamma_{2}\right)^{2}} \\
\text { Substituting } \begin{array}{r}
q_{M}^{d d}, w_{M}^{d d} \text { into } \lambda_{M}^{d d}, R(t) \\
\lambda_{M}^{d d}=\frac{k_{1} \gamma_{2}\left(\alpha+\beta\left(\Delta \tau-c_{m}\right)\right)-k_{1} \gamma_{2}^{2} R}{4 \beta k_{1}-\left(\gamma_{1}+\gamma_{2}\right)^{2}} \\
+\frac{4 \beta k_{1}-\gamma_{1}^{2}-\gamma_{1} \gamma_{2}}{4 \beta k_{1}-\left(\gamma_{1}+\gamma_{2}\right)^{2}} \theta \lambda_{M}^{\text {dd }}
\end{array}
\end{array}
$$

$$
\begin{aligned}
& \dot{R(t)}=\frac{4 \beta \theta^{2} \lambda_{M}^{d d}+\left(\gamma_{1}+\gamma_{2}\right) \theta\left(\alpha+\beta\left(\Delta \tau-c_{m}\right)\right.}{4 \beta k_{1}-\left(\gamma_{1}+\gamma_{2}\right)^{2}} \\
& -\frac{4 \beta k_{1}-\gamma_{1}^{2}-\gamma_{1} \gamma_{2}}{4 \beta k_{1}-\left(\gamma_{1}+\gamma_{2}\right)^{2}} \theta R
\end{aligned}
$$

Rewriting them, we get:

$$
\left[\begin{array}{c}
\dot{d} d \\
\dot{R}
\end{array}\right]=A\left[\begin{array}{c}
\lambda_{M}^{d d} \\
R
\end{array}\right]+b
$$

where 


$$
A=\left[\begin{array}{cc}
\frac{4 \beta k_{1}-\gamma_{1}^{2}-\gamma_{1} \gamma_{2}}{4 \beta k_{1}-\left(\gamma_{1}+\gamma_{2}\right)^{2}} \theta & \frac{-k_{1} \gamma_{2}^{2}}{4 \beta k_{1}-\left(\gamma_{1}+\gamma_{2}\right)^{2}} \\
\frac{4 \beta \theta^{2}}{4 \beta k_{1}-\left(\gamma_{1}+\gamma_{2}\right)^{2}} & -\frac{4 \beta k_{1}-\gamma_{1}^{2}-\gamma_{1} \gamma_{2}}{4 \beta k_{1}-\left(\gamma_{1}+\gamma_{2}\right)^{2}} \theta
\end{array}\right]
$$

and

$$
b=\left[\begin{array}{c}
\frac{k_{1} \gamma_{2}\left(\alpha+\beta\left(\Delta \tau-c_{m}\right)\right)}{4 \beta k_{1}-\left(\gamma_{1}+\gamma_{2}\right)^{2}} \\
\frac{\left(\gamma_{1}+\gamma_{2}\right) \theta\left(\alpha+\beta\left(\Delta \tau-c_{m}\right)\right)}{4 \beta k_{1}-\left(\gamma_{1}+\gamma_{2}\right)^{2}}
\end{array}\right]
$$

The eigenvalues of A are given by

$$
\begin{array}{r}
{\left[\begin{array}{l}
r_{1} \\
r_{2}
\end{array}\right]=\left[\begin{array}{c}
\frac{\theta \sqrt{N_{1}^{2}-4 k_{1} \gamma_{2}^{2} \beta}}{4 k_{1} \beta-\left(\gamma_{1}+\gamma_{2}\right)^{2}} \\
\frac{\theta \sqrt{N_{1}^{2}-4 k_{1} \gamma_{2}^{2} \beta}}{4 k_{1} \beta-\left(\gamma_{1}+\gamma_{2}\right)^{2}}
\end{array}\right]} \\
N_{1}=4 k_{1} \beta-\gamma_{1}^{2}-\gamma_{1} \gamma_{2}
\end{array}
$$

is:

and the corresponding matrix of eigenvectors of $A$

$$
P=\left[\begin{array}{cc}
P_{1} & P_{2} \\
1 & 1
\end{array}\right]
$$

Where

$$
P_{1}=\frac{N_{1}+\sqrt{N_{1}^{2}-4 k_{1} \gamma_{2}^{2} \beta}}{4 \beta \theta}, P_{2}=\frac{N_{1}-\sqrt{N_{1}^{2}-4 k_{1} \gamma_{2}^{2} \beta}}{4 \beta \theta}
$$

Thus, we have:

$$
\left[\begin{array}{c}
\lambda_{R}^{d d} \\
R
\end{array}\right]=P\left[\begin{array}{cc}
e^{r^{3 t}} & 0 \\
0 & e^{r_{t} t}
\end{array}\right]\left[\begin{array}{l}
k_{1} \\
k_{2}
\end{array}\right]-A^{-1} b
$$

According to the boundary conditions condition:

$$
R^{d d}(0)=R_{0} \quad \text { and } \quad \lambda_{M}^{d d}(T)=0
$$

the analytical solutions of $R^{d d}(t), \lambda_{M}^{\text {dd }}(t)$ can be acquired as:

$$
\begin{array}{r}
R^{d d}(t)=h_{1} e^{r_{1} t}+h_{2} e^{r_{2} t}-B_{1} \\
\lambda_{M}^{d d}(t)=h_{1} \frac{N_{1}+\sqrt{N_{1}^{2}-4 k_{1} \gamma_{2}^{2} \beta}}{4 \beta \theta} e^{r_{1} t} \\
+h_{2} \frac{N_{1}-\sqrt{N_{1}^{2}-4 k_{1} \gamma_{2}^{2} \beta}}{4 \beta \theta} e^{r_{2} t}-A_{1}
\end{array}
$$

Where

$$
\begin{gathered}
A_{1}=\frac{k_{1}\left(\gamma_{2}^{2}\left(\gamma_{1}+\gamma_{2}\right)-N_{1} \gamma_{2}\right)\left(\alpha+\beta\left(\Delta \tau-c_{m}\right)\right)}{\theta\left(4 \beta k_{1} \gamma_{2}^{2}-N_{1}^{2}\right)} \\
B_{1}=\frac{\left(N_{1}\left(\gamma_{1}+\gamma_{2}\right)-4 \beta k_{1} \gamma_{2}\right)\left(\alpha+\beta\left(\Delta \tau-c_{m}\right)\right)}{4 \beta k_{1} \gamma_{2}^{2}-N_{1}^{2}} \\
h_{1}=\frac{\left(R_{0}+B_{1}\right)\left(N_{1}-\sqrt{\left.N_{1}^{2}-4 k_{1} \gamma_{2}^{2} \beta\right)} e^{r_{2} T}-4 \beta \theta A_{1}\right.}{\left(N_{1}-\sqrt{N_{1}^{2}-4 k_{1} \gamma_{2}^{2} \beta}\right) e^{r^{2} T}-\left(N_{1}+\sqrt{N_{1}^{2}-4 k_{1} \gamma_{2}^{2} \beta}\right) e^{r_{1} T}}
\end{gathered}
$$

$$
\begin{gathered}
h_{2}=\frac{\left(R_{0}+B_{1}\right)\left(N_{1}+\sqrt{N_{1}^{2}-4 k_{1} \gamma_{2}^{2} \beta}\right) e^{r^{r T}}-4 \theta \beta A_{1}}{\left(N_{1}+\sqrt{N_{1}^{2}-4 k_{1} \gamma_{2}^{2} \beta}\right) e^{r_{1} T}-\left(N_{1}-\sqrt{N_{1}^{2}-4 k_{1} \gamma_{2}^{2} \beta}\right) e^{r_{2} T}} \\
\text { Substituting } R^{d d}(t), \lambda_{M}^{d d}(t) \text {, then we can get the }
\end{gathered}
$$
equilibrium whosesale price, retail price, quality strategy respectively. The proof is complete. 